Document downloaded from:

http://hdl.handle.net/10251/51140

This paper must be cited as:

Lago, MA.; Rupérez Moreno, MJ.; Martínez Martínez, F.; Monserrat Aranda, C.; Larra, E.; Gueell, JL.; Peris-Martinez, C. (2015). A new methodology for the in vivo estimation of the elastic constants that characterize the patient-specific biomechanical behavior of the human cornea. Journal of Biomechanics. 48(1):38-43. doi:10.1016/j.jbiomech.2014.11.009.

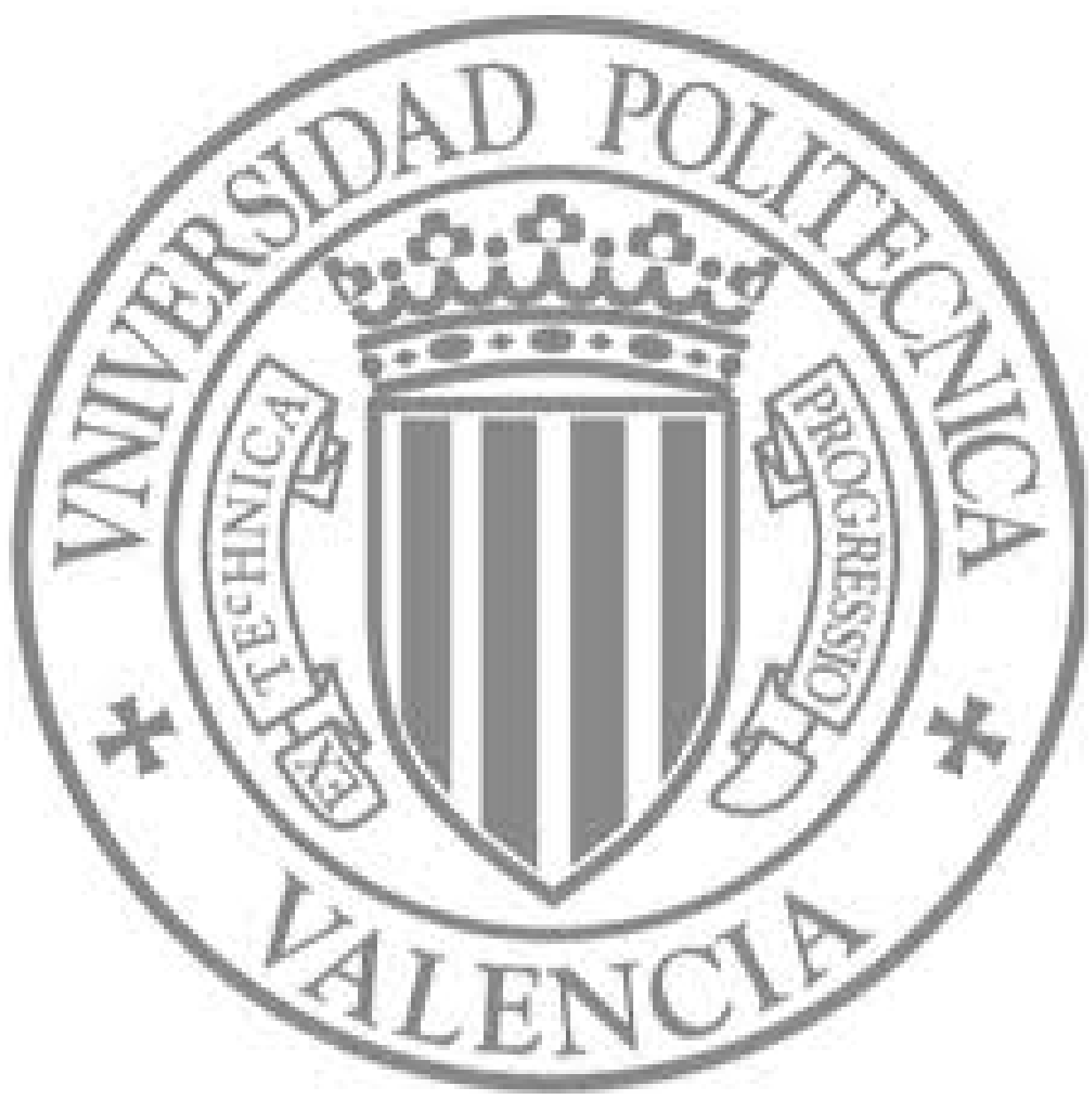

The final publication is available at

http://dx.doi.org/10.1016/j.jbiomech.2014.11.009

Copyright Elsevier 


\title{
A new methodology for the in-vivo estimation of the elastic constants that characterize the patient-specific biomechanical behavior of the human cornea
}

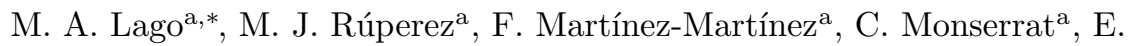 \\ Larra $^{\mathrm{b}}$, J. L. Güell ${ }^{\mathrm{c}}$, C. Peris-Martínez ${ }^{\mathrm{d}}$ \\ ${ }^{a}$ LabHuman, Universitat Politècnica de València, Camino de Vera s/n, 46022, Valencia, \\ Spain \\ ${ }^{b} A J L$ Ophtalmics \\ ${ }^{c}$ Instituto de Microcirugía Ocular \\ ${ }^{d}$ Fundación Oftalmológica del Mediterráneo
}

\begin{abstract}
This work presents a methodology for the in-vivo characterization of the complete biomechanical behavior of the human cornea of each patient. Specifically, the elastic constants of a hyperelastic, second-order Ogden model were estimated for 24 corneas corresponding to 12 patients. The finite element method was applied to simulate the deformation of human corneas due to non-contact tonometry, and an iterative search controlled by a genetic heuristic was used to estimate the elastic parameters that most closely approximates the simulated deformation to the real one. The results from a synthetic experiment showed that these parameters can be estimated with an error of about $5 \%$. The results of 24 in-vivo corneas showed an overlap of about $90 \%$ between simulation and real deformed cornea and a modified Hausdorff distance of $25 \mu \mathrm{m}$, which indicates the great accuracy of the proposed methodology.
\end{abstract}

Keywords: biomechanical characterization, parameter optimization, cornea

\section{Introduction}

The biomechanical characterization of in-vivo tissue is a growing field in surgical planning. Being able to characterize the mechanical behavior of human organs may allow the clinician to simulate their response to any manipulation performed by surgeons during an intervention. The case of the cornea is especially complex since the cornea shows a great variability between individuals and also has a strong sensitivity to age or temperature, thus conditioning its biomechanical behavior [6]. Being able to estimate in-vivo the mechanical response

\footnotetext{
* Corresponding author

Email address: malago@labhuman.com (M. A. Lago)
} 
of the cornea can allow the detection of some pathologies whose symptoms may change the stiffness of the cornea [10]. Furthermore, the in-vivo estimation of the mechanical behavior of the cornea is the core of the analysis of the real response during surgical interventions $[2,4,15,7,20]$.

A biomechanical model of the cornea describes the response of the cornea in terms of displacements to the application of loads and the model can be used to simulate how the curvature of the cornea recovers after the implantation of intracorneal segments in patients with keratoconus [5]. In [2], the authors stated that a biomechanical study before refractive corneal surgery is very helpful in quantitatively assessing the effect of each parameter on the optical outcome. In their work, a mechanical model of the human cornea was proposed and implemented under a finite element context to simulate the effects of some common surgical procedures such as photorefractive keratectomy (PRK) and limbal relaxing incisions (LRI). The model considered a non-linear anisotropic hyperelastic behavior of the cornea. The authors evaluated the effect of the incision variables on the change of curvature of the cornea to correct myopia and astigmatism. They concluded that the model reasonably approximated the corneal response to an increasing pressure. They also showed that tonometry underestimates the value of the IOP (Intra Ocular Pressure) after photorefractive keratectomy (PRK) or LASIK surgery.

The human cornea is considered to be an anisotropic, hyperelastic, and incompressible tissue [6]. Many different biomechanical models have been presented in the literature to model cornea behavior [12, 11, 15, 19, 20, 22]. Most previous works measured the elastic constants of those models using ex-vivo human corneas from an eye bank $[23,6]$. Others, however, have used the material properties of animal corneas $[1,3]$. Even though all of the constitutive models proposed in the literature up to now may be physically correct for modeling the mechanical behavior of the cornea, their accuracy (the realism provided by the simulation of the mechanical behavior of the cornea) relies on the elastic constants of those models, which for now are only an approximation of their real values. They are not patient-specific or estimated in-vivo. In order to get an accurate biomechanical model of the cornea, its mechanical behavior must be patient-specific and characterized in-vivo.

The ORA (Ocular Response Analyzer) device is one of the most commonly known devices for the characterization of corneal biomechanics in ophthalmology. This device provides the IOP along with the corneal hysteresis and the corneal resistance factor, which are a representation of the viscoelasticity of the cornea. The ORA results have been applied to obtain the relationship among the hysteresis, IOP, glaucoma, keratoconus, or aging [16, 13, 21]. However, the parameters provided by this device are not able to construct a physical model that is capable of reproducing the above-mentioned physical deformations; they are only an indirect measure of the elasticity of the cornea. In [8], the ORA device was used to construct a viscoelastic biomechanical model of the cornea. By attaching a camera to the device, the authors validated the proposed model using a contact lens as a cornea model. However, as the authors stated, the proposed methodology was able to determine changes or variability in the elas- 
ticity of the cornea, but it could not estimate the biomechanical behavior of the cornea.

In 2011, the OCULUS Corvis ${ }^{\circledR}$ ST (OCULUS Optikgeräte GmbH, Münchholzhäuser Str. 29 D-35582 Wetzlar, Germany) was presented. This tonometer and pachymeter also incorporates a high-speed camera that records the cornea when applying a controlled force by an air jet. Additionally, it provides the images of the 1st and 2nd applanation of the cornea in conjuntion with the ultrahigh-speed video of the corneal deformation. The Corvis ST has been approved by the US Food and Drug Administration as a tonometer and pachymeter but not for biomechanical applications. Nevertheless, the images and the video provided by this device can be used to obtain the in-vivo biomechanical response of the human cornea. Therefore, the elastic constants that characterize the constitutive equations of the models proposed for the human cornea can be estimated for each patient.

This paper presents a methodology to estimate the elastic constants that characterize the constitutive equations that describe the in-vivo biomechanical behavior of the human cornea for each patient. The Corvis ST device was used to obtain a video sequence of the cornea deformation. Using the images of the deformed cornea from this sequence, a search algorithm iterates over the elastic constants in order to simulate a deformation that is as close as possible to the real deformed cornea. The methodology was applied to 24 different corneas from 12 volunteer patients in order to estimate the hyperelastic behavior of the corneal tissue specifically for each eye of each patient. To the authors' knowledge, this is the first time that the elastic constants that describe the biomechanical behavior of the human cornea are characterized in-vivo.

\section{Materials and methods}

The methodology presented in this paper simulates the deformation of the cornea when applying an air jet to measure the IOP. This process is known as non-contact tonometry. The Corvis ST device was used by the Fundación Oftalmológica del Mediterráneo to take images of the cornea during the air pressure loading, thus obtaining a sequence of images from the initial state, passing through the state of maximum deformation, and eventually ending in an undeformed state. The simulation of the cornea deformation was performed using the Finite Element Method (FEM), which has been proved to be suitable for large deformations $[8,15]$. Both segmentation and meshing of the cornea were performed using a MATLAB ${ }^{\circledR}$ script, which also controlled the subsequent iterative search algorithm.

\subsection{Biomechanical model and boundary conditions}

In this work, a second-order hyperelastic Ogden model was chosen since it provides enough complexity to prove the methodology without disregarding its realism. The energy potential function of the N-order Ogden model is defined in Equation 1. 


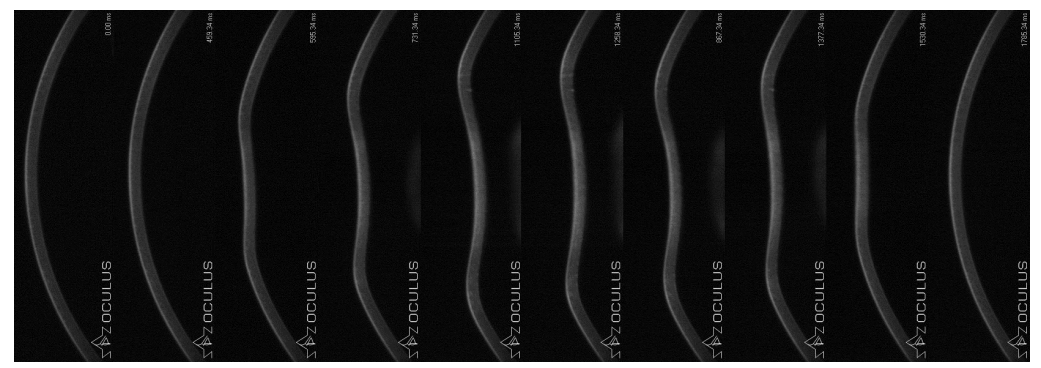

Figure 1: Video sequence taken by the Corvis ST high-speed camera.

$$
W_{O}=\sum_{i=1}^{N} \frac{\mu_{i}}{\alpha_{i}}\left(\bar{\lambda}_{1}^{\alpha_{i}}+\bar{\lambda}_{2}^{\alpha_{i}}+\bar{\lambda}_{3}^{\alpha_{i}}-3\right)+\sum_{i=1}^{N} \frac{K_{0_{i}}}{2}(J-1)^{2 i}
$$

where $N$ stands for the order; $\mu_{i}$ and $\alpha_{i}$ stand for the elastic parameters; $\bar{\lambda}_{1}$, $\bar{\lambda}_{2}$, and $\bar{\lambda}_{3}$ are the deviatoric stretches; $K_{0_{i}}$ is the initial Bulk modulus; and $J$ is the determinant of the elastic deformation gradient. The $K_{0_{i}}$ coefficient was fixed to $10^{7} \mathrm{~Pa}$ assuming that the cornea is incompressible [15]. Therefore, for a second-order Ogden model, the parameters to be estimated in the model are the following: $\left\langle\mu_{1}, \alpha_{1}, \mu_{2}, \alpha_{2}\right\rangle$.

The pictures taken from the Corvis ST device correspond to a 2D slice of a sectional plane of the real cornea of each patient. A light beam falls upon this plane during the air jet, and the cornea scatters the light reflecting the sectional plane on the camera sensor at an angle of $45^{\circ}$. Figure 1 shows some frames of the high-speed video sequence taken from the device.

A 2D finite element mesh was constructed from the initial 2D slice using triangular elements. The nodes of the mesh corresponding to the edge of the peripheral cornea were restricted in all directions representing the anchoring with the sclera, which is thought to be 5 times stiffer than the cornea. The air jet was applied at the apex of the cornea with an average force of $15 \mathrm{mmHg}$ and an estimated diameter of $3 \mathrm{~mm}$, according to the manufacturer's indications.

Additionally, The state of maximum deformation of the cornea was taken from the high-speed video after the air jet application. The deformed cornea was segmented in order to compare the biomechanical simulation of its deformation with the real one.

Since the initial state of the cornea is actually subjected to the IOP and cannot be considered to be an undeformed state, the first step is to reproduce the deformed state due to the deformation caused by this pressure by estimating the initial stresses to which the cornea is subjected. This process is independent of the tonometer used to measure the IOP. It is also independent of the deformation of the cornea caused by the air jet produced by the Corvis ST device, and it is performed before its simulation. After the segmentation of the deformed cornea, the initial stresses were extracted by an iterative algorithm within the parameter search. The application of the initial stresses on the biomechanical 


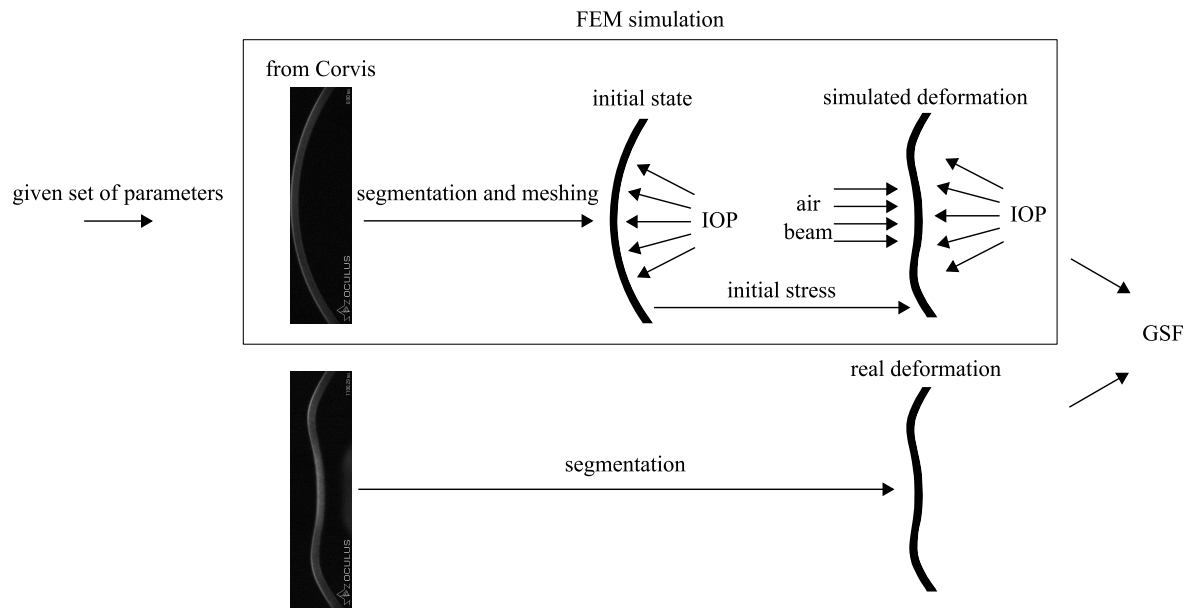

Figure 2: The image of the cornea is segmented and meshed in both deformed and undeformed status. The IOP is applied to the undeformed model in order to obtain the initial stresses that are used to simulate the deformation due to the air jet. Finally, the simulated deformation is compared with the real deformation of the cornea, and the GSF value is retrieved. This is performed for each candidate set of parameters in the iterative algorithm.

model resulted in the initial state of the cornea. Then, the air jet was applied at the center of the cornea with the corresponding force and a new FE simulation was performed. This was carried out for each set of parameters proposed by the search algorithm.

\subsection{Search algorithm}

Generally, the optimization of a function is often performed by iterative search algorithms. These algorithms work by changing the input parameters of a fitness function $(\mathbf{X})$ in an attempt to to minimize (or maximize) its values as Eq. 2 shows.

$$
\hat{\mathbf{X}}=\arg \min f(\mathbf{X}) \quad \text { where } \mathbf{X}=\left\{x_{1}, x_{2}, \cdots, x_{n}\right\}
$$

In this case, the function to optimize was the dissimilarity of the simulated deformed cornea and the real deformed cornea. The difference was calculated using the Geometric Similarity Function (GSF) which is a combination of the Jaccard Coefficient (JC) and the Modified Hausdorff Coefficient (MHD) [14]. JC measures the overlap of two volumes with 1 being a perfect overlap and 0 being no overlap at all. MHD is the average of the maximum distances between the surfaces of two volumes. Thus, the GSF takes into account not only the overlap of the volumes but also their absolute distance. Therefore, it is a suitable function to be optimized in order to find the elastic parameters [18].

$$
G S F=\log ((1-J C) M H D)
$$


Since the GSF may present local minima (or maxima), complex algorithms need to be used to minimize its value. In this work, a genetic algorithm drives the iterative search. Genetic heuristics have proved to be suitable to estimate the elastic constants of soft tissue [9, 17], achieving errors of less than $4 \%$.

This methodology for estimating the parameters of the model has three steps: Initialization, Generation and Termination. Figure 2 shows the generation step for each candidate set of parameters.

1. Initialization: the algorithm generates an initial random population of elastic parameters $\mathbf{X}_{0}$ within a given interval for each parameter. Additionally, the real deformed cornea is segmented.

2. Generation: successive populations $\mathbf{X}_{i+1}$ are generated iteratively by means of the following steps:

i. Initial stress is calculated for each individual set of parameters as follows:

a. The IOP is applied to the internal surface of the cornea considering its original state as a non-deformed state.

b. The FEM simulates this loaded state with the Ogden model and the set of elastic constants estimated in each case by the search algorithm. This way, the stresses in the cornea are extracted.

c. The extracted stresses are applied to the cornea as initial stresses along with the IOP.

d. Steps a. and b. are iterated until the displacement of the cornea is negligible.

e. The final stresses are obtained and used as the initial stress state for further deformation.

ii. The air jet is applied to the model with the initial stress, and the FEM is solved using each individual set of parameters.

iii. The GSF between each simulated deformation of the cornea and the real deformation is evaluated in the current population.

iv. The best individual sets are selected and tagged as parents.

v. A new population is generated both by combining parents (crossover) or by changing a parent randomly (mutation).

3. Termination: step 2 is iterated until a stop condition is triggered.

The stop condition can be from a maximum number of iterations, a sensitivity test between two iterations, or a timer. The parameters that achieve the best GSF value (which corresponds to the deformation that is most similar to the real one) are designated as the estimated parameters of the biomechanical model $\hat{\mathbf{X}}$. 
Table 1: Target parameters (Tg.), estimated parameters (Est.), and mean relative error (RE) for the synthetic experiment.

\begin{tabular}{|c|c|c|c|c|c|c|c|c|}
\hline & $\begin{array}{c}\mathrm{IOP} \\
(\mathrm{mmHg})\end{array}$ & $\begin{array}{c}\text { Pachy. } \\
(\mu \mathrm{m})\end{array}$ & $\begin{array}{c}\mu_{1} \\
(\mathrm{~Pa})\end{array}$ & $\alpha_{1}$ & $\begin{array}{c}\mu_{2} \\
(\mathrm{~Pa})\end{array}$ & $\alpha_{2}$ & $\mathrm{JC}$ & $\begin{array}{l}\text { MHD } \\
(\mu \mathrm{m})\end{array}$ \\
\hline Tg. $\mathbf{X}_{\mathbf{1}}$ & \multirow{2}{*}{10.5} & \multirow{2}{*}{595} & 11000 & 30 & 35000 & 80 & - & - \\
\hline Est. $\hat{\mathbf{X}}_{1}$ & & & 10711 & 31.32 & 33751 & 82.44 & 0.99 & 4.6 \\
\hline $\mathrm{RE}(\%)$ & - & - & 2.63 & 4.4 & 3.6 & 3.05 & - & - \\
\hline Tg. $\mathbf{X}_{\mathbf{2}}$ & \multirow{2}{*}{10.5} & \multirow{2}{*}{595} & 36000 & 50 & 32500 & 88 & - & - \\
\hline Est. $\hat{\mathbf{X}}_{\mathbf{2}}$ & & & 34031 & 50.07 & 32694 & 89.71 & 0.99 & 4.8 \\
\hline $\mathrm{RE}(\%)$ & - & - & 5.47 & 0.14 & 0.6 & 1.94 & - & - \\
\hline $\begin{array}{c}\text { Mean } \\
\operatorname{RE}(\%)\end{array}$ & - & - & 4.05 & 2.27 & 2.1 & 2.5 & - & - \\
\hline
\end{tabular}

\section{Results}

First, a synthetic experiment was performed in order to probe the methodology. The same cornea was deformed with two target sets of elastic constants of the biomechanical model. The target sets were $\mathbf{X}_{1}:\left\langle\mu_{1}=11000, \alpha_{1}=30, \mu_{2}=\right.$ $\left.35000, \alpha_{2}=80\right\rangle$ and $\mathbf{X}_{2}:\left\langle\mu_{1}=36000, \alpha_{1}=50, \mu_{2}=32500, \alpha_{2}=88\right\rangle$. Their selection was arbitrary within a previously estimated interval. The selection of IOP and pachymetry was also arbitrary, with the values of these variables corresponding to an average value among the values for all the patients. The search algorithm was launched in order to find these given sets of parameters. The results are shown in Table 1.

Afterward, 24 samples from 12 patients with healthy corneas between the ages of 25 and 40 years old were used to apply the methodology. These patients were volunteers who gave informed consent. The deformations of the corneas were registered by the Corvis ST device in the Fundación Oftalmológica del Mediterráneo, and the specific value of the IOP and the pachymetry were measured for each cornea.

The biomechanical behavior of the 24 corneas was characterized, estimating the elastic constants of each one for the second-order Ogden model. Table 2 shows the estimated parameters for the left and right corneas for all of the patients as well as the JC and MHD values.

Figure 3 shows the original segmented cornea, the simulated deformed cornea, and their differences for one patient. The gray values identify the correspondence between the two segmentations while the black and white pixels represent the signed differences (positive and negative). 
Table 2: Estimated biomechancial constants for Left and Right eyes for each patient.

\begin{tabular}{ccccccccc}
\hline Patient & $\begin{array}{c}\text { IOP } \\
(\mathrm{mmHg})\end{array}$ & $\begin{array}{c}\text { Pachy. } \\
(\mu \mathrm{m})\end{array}$ & $\begin{array}{c}\mu_{1} \\
(\mathrm{~Pa})\end{array}$ & $\alpha_{1}$ & $\begin{array}{c}\mu_{2} \\
(\mathrm{~Pa})\end{array}$ & $\alpha_{2}$ & $\mathrm{JC}$ & $\begin{array}{c}\mathrm{MHD} \\
(\mu \mathrm{m})\end{array}$ \\
\hline \multirow{2}{*}{$\mathbf{P}_{1}$} & 15 & 533 & 36508 & 71.62 & 13220 & 50.17 & 0.88 & 22.6 \\
& 12 & 535 & 25239 & 74.73 & 15360 & 79.10 & 0.88 & 23 \\
\hline \multirow{2}{*}{$\mathbf{P}_{2}$} & 11.5 & 499 & 38160 & 91.73 & 29536 & 83.21 & 0.87 & 21.2 \\
& 11 & 520 & 27220 & 82.29 & 31040 & 90.24 & 0.87 & 23 \\
\hline \multirow{2}{*}{$\mathbf{P}_{3}$} & 8.5 & 458 & 27261 & 91.06 & 29539 & 46.53 & 0.84 & 30.8 \\
& 8.5 & 479 & 39492 & 96.80 & 14822 & 6.13 & 0.87 & 24.4 \\
\hline \multirow{2}{*}{$\mathbf{P}_{4}$} & 12.5 & 520 & 30434 & 92.28 & 7327 & 32.74 & 0.88 & 25 \\
& 8.5 & 524 & 29588 & 86.80 & 31693 & 4.37 & 0.89 & 19.8 \\
\hline \multirow{2}{*}{$\mathbf{P}_{5}$} & 12 & 580 & 38885 & 77.89 & 29141 & 60.15 & 0.83 & 31.4 \\
& 11.5 & 601 & 22120 & 98.86 & 13096 & 91.94 & 0.82 & 32.2 \\
\hline \multirow{2}{*}{$\mathbf{P}_{6}$} & 14 & 567 & 24495 & 33.37 & 19519 & 80.66 & 0.88 & 21.6 \\
& 17 & 568 & 22546 & 74.73 & 16950 & 80.65 & 0.87 & 23 \\
\hline \multirow{2}{*}{$\mathbf{P}_{7}$} & 11 & 527 & 10978 & 31.38 & 33946 & 79.82 & 0.88 & 21 \\
& 12 & 540 & 22921 & 68.02 & 20739 & 70.84 & 0.88 & 21 \\
\hline \multirow{2}{*}{$\mathbf{P}_{8}$} & 10.5 & 595 & 39721 & 60.32 & 14361 & 69.21 & 0.90 & 19.8 \\
& 9 & 588 & 27156 & 18.03 & 38305 & 80.82 & 0.89 & 20.6 \\
\hline \multirow{2}{*}{$\mathbf{P}_{9}$} & 13 & 606 & 17503 & 91.11 & 32674 & 53.86 & 0.89 & 21.4 \\
& 14 & 613 & 23161 & 91.77 & 3731 & 78.22 & 0.89 & 21 \\
\hline \multirow{2}{*}{$\mathbf{P}_{10}$} & 13.5 & 584 & 26511 & 84.26 & 23502 & 37.52 & 0.90 & 20.6 \\
& 12.5 & 596 & 5119 & 97.99 & 34767 & 56.81 & 0.89 & 19.8 \\
\hline \multirow{2}{*}{$\mathbf{P}_{11}$} & 8 & 513 & 8458 & 98.14 & 27897 & 91.71 & 0.80 & 41 \\
& 8.5 & 522 & 30704 & 98.98 & 15862 & 43.92 & 0.79 & 43.2 \\
\hline \multirow{2}{*}{$\mathbf{P}_{12}$} & 12.5 & 558 & 12330 & 79.19 & 21224 & 83.78 & 0.83 & 35.8 \\
& 14.5 & 555 & 38327 & 11.27 & 32852 & 76.47 & 0.88 & 24.4 \\
\hline \hline \multirow{2}{*}{ Avg. $\hat{\mathbf{X}}$} & & & 26034 & 75.11 & 22962 & 63.70 & 0.87 & 25.4 \\
Std.Dev. & & & 9960 & 26.03 & 9531 & 25.01 & 0.03 & 6.8 \\
\hline & & & & & & & &
\end{tabular}




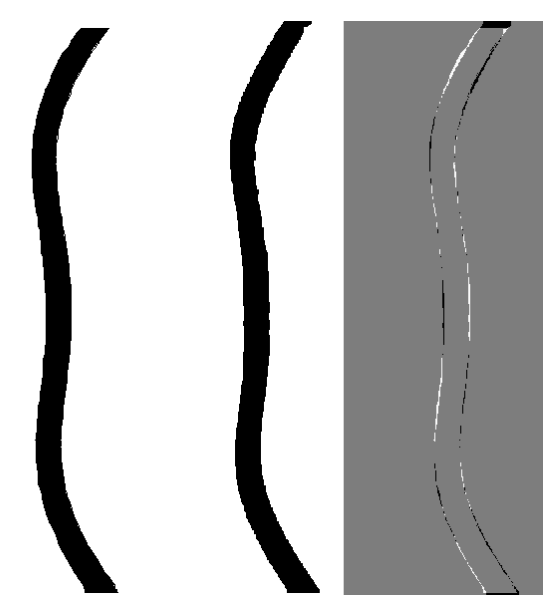

Figure 3: Left: real deformation of the cornea when applying the air jet. Center: simulated deformation of the cornea when applying the air jet. Right: signed difference of the original and simulated cornea, the gray values are identical in both images; the black and white values are signed differences)

\section{Discussion}

The estimated parameters for the synthetic cases proved that the methodology is able to obtain a biomechanical model that is very similar to the target one. A mean relative error of less than $5 \%$ in the estimation of the $\mu$ parameters and an error of around $2.5 \%$ in the estimation of $\alpha$ parameters indicate a very small deviation from the target parameters. Even though GSF is very useful for performing the iterative search since it considers both overlap and distance, it is hard to translate its values into a real meaning. Therefore, only JC and MHD values are used to interpret the results. JC showed an overlap of $99 \%$, and MHD showed a maximum distance of $2.5 \mu \mathrm{m}$.

Moving on to the experiment with real corneas, the differences between pixels observed in Figure 3 show a thin line of non-matching pixels along the surface of the cornea (internal and external). Table 1 shows that this distribution of nonmatching pixels led to $\mathrm{JC}$ values of around 0.87 ( $87 \%$ overlap) which means that the majority of the cornea tissue is overlapping. Additionally, having as many white pixels as black pixels indicates that the maladjustment is distributed all along the surface and in the same proportion. On the other hand, the MHD values showed that the distances between the simulated and real deformed cornea were, on average, close to near $25 \mu \mathrm{m}$ ( $5 \%$ of the average corneal thickness), which indicates the high resemblance of the two images. Additionally, the JC and MHD values allow knowing how good the adjustment is, which gives an idea about the reliability of the estimated model for that specific patient.

The estimated parameters of the Ogden model obtained between individual patients proved to be very different due to the high variability in composition and heterogeneity of the population. Average values of $26034 \pm 9960 \mathrm{~Pa}$ and 
$22962 \pm 9531 \mathrm{~Pa}$ for $\mu_{1}$ and $\mu_{2}$, respectively, and $75.11 \pm 26.03$ and $63.70 \pm 25.01$ for $\alpha_{1}$ and $\alpha_{2}$, respectively, are in consonance with previous studies that pointed out this variability [6].

Even though an isotropic hyperelastic Ogden model was used to prove the algorithm, the methodology presented here can be applied to any other biomechanical model chosen to represent the behavior of the cornea, even adding more complexity like considering anisotropy or viscoelasticity. The only difference will be the number of parameters that the genetic algorithm has to manage and the consequent computation time necessary.

The Corvis ST device that was used for the image acquisition proved to be suitable for this methodology even though more complex personalized devices could be used to characterize the anisotropy or viscoelasticity of the model. To the authors' knowledge, this is the only device currently on the market that can provide real-time video of the deformation, which allows the in-vivo characterization of the behavior of the human cornea. If more information about the undeformed and deformed cornea (such as a 3D reconstruction or a different type of deformation) were available, this methodology could achieve a much more accurate model.

The IOP determined by the tonometer Corvis ST is used to establish the initial state of the cornea. This is not a undeformed state that is free of loads but rather a state of initial stress which must be determined since it is an input to the model. According to most ophthalmologists, there is currently no clinical device that actually measures this variable accurately, and the methodology described here to estimate this initial stress state is independent of the device used to measure the IOP. This variable influences the accuracy of the estimated biomechanical model.

The worst values of JC and MHD appeared in cases where the position of the air jet was not exactly aimed at the center of the cornea, while, in the simulated model, it is always applied at its center. Therefore, the image acquisition must be carefully performed in order to avoid inaccurate and unreliable results. Correcting these abnormalities can lead to higher values of JC (similar to those obtained in the synthetic experiments), which would guarantee a lower error in the parameter estimation.

This methodology proved to be suitable in a simple 2D geometrical model of the cornea. Having a 3D geometry of the cornea which takes into account the thickness variability from the center to the periphery would increase the accuracy of the estimated biomechanical model and it would broaden the applications of having a biomechanical characterization of the corneal tissue. These $3 \mathrm{D}$ geometries of the cornea can be obtained by devices to measure the topography such as Pentacam.

It is important to highlight that the aim of the methodology presented in this paper is to characterize the in-vivo mechanical behavior of the corneal tissue of each patient. The main goal is to construct a model that accurately simulates the deformation that the cornea would undergo during a manipulation in a refractive surgery procedure. This methodology describes how to construct an accurate physical model for the cornea, and, therefore, no physiological parame- 
ters were estimated. In this paper, it has been proved that this can be routinely done within the clinical protocol and that the in-vivo biomechanical behavior of the cornea can be characterized specifically for each patient. Moreover, with these simulated deformations, additional physiological parameters could also be inferred.

\section{Conclusions}

This paper presented a methodology for the in-vivo estimation of the elastic constants that characterize the biomechanical behavior of the cornea of each patient. A search based on genetic heuristics was developed and the parameters of a hyperelastic, second-order Ogden model were estimated for each patient. Twenty-four patient-specific biomechanical models were constructed which proved to be very similar to the real behavior of the patients' corneas. This methodology is easily applicable to any biomechanical model and could be applied to more complex models also adding anisotropy and viscoelasticity. Furthermore, being able to estimate the biomechanical behavior of the cornea would also allow physiological and optical parameters like hysteresis or refractive power to be inferred and would also allow the cornea response to be simulated

during and after refractive surgical procedures. Additionally, a very interesting line of research would be to classify cornea behavior taking into account different factors such as gender, age, or ocular diseases.

\section{Conflict of interest statement}

The authors of this manuscript have no conflict of interest with the presented work.

\section{Acknowledgements}

This project has been partially funded by MECD (reference AP2009-2414) and MINECO (INNPACTO, IPT-2012-0495-300000).

[1] Ahearne, M., Yang, Y., Then, K., Liu, K.-K., 2007. An indentation technique to characterize the mechanical and viscoelastic properties of human and porcine corneas. Annals of Biomedical Engineering 35 (9), 1608-1616.

[2] Alastrué, V., Calvo, B., Pena, E., Doblaré, M., 2006. Biomechanical modeling of refractive corneal surgery. Journal of biomechanical engineering 128 (1), 150-160.

[3] Boyce, B., Jones, R., Nguyen, T., Grazier, J., 2007. Stress-controlled viscoelastic tensile response of bovine cornea. Journal of Biomechanics 40 (11), $2367-2376$. 
[4] Deenadayalu, C., Mobasher, B., Rajan, S., Hall, G., 2006. Refractive change induced by the LASIK flap in a biomechanical finite element model. J Refract Surg 22 (3), 286-292.

[5] delBuey, M., Lanchares, E., Cristobal, J., Calvo, B., Lavilla, L., 2010. Comparative biomechanical analysis of combined treatments for keratoconus. In proceedings of ASCRS Symposium and Congress, Boston, MA.

[6] Elsheikh, A., Wang, D., Brown, M., Rama, P., Campanelli, M., Pye, D., 2007. Assessment of corneal biomechanical properties and their variation with age. Current Eye Research 32 (1), 11-19.

[7] Gefen, A., Shalom, R., Elad, D., Mandel, Y., 2009. Biomechanical analysis of the keratoconic cornea. Journal of the Mechanical Behavior of Biomedical Materials 2 (3), $224-236$.

[8] Glass, D. H., Roberts, C. J., Litsky, A. S., Weber, P. A., 2008. A viscoelastic biomechanical model of the cornea describing the effect of viscosity and elasticity on hysteresis. Investigative Ophthalmology and Visual Science 49 (9), 3919-3926.

[9] Harb, N., Labed, N., Domaszewski, M., Peyraut, F., 2011. A new parameter identification method of soft biological tissue combining genetic algorithm with analytical optimization. Computer Methods in Applied Mechanics and Engineering 200 (14), $208-215$.

[10] Hjortdal, J. Ø., 1995. Extensibility of the normo-hydrated human cornea. Acta Ophthalmologica Scandinavica 73 (1), 12-17.

[11] Jr., W. J. D., Wilson, S. E., 2006. Biomechanics and wound healing in the cornea. Experimental Eye Research 83 (4), 709 - 720.

[12] Kaliske, M., 2000. A formulation of elasticity and viscoelasticity for fibre reinforced material at small and finite strains. Computer Methods in Applied Mechanics and Engineering 185 (24), 225 - 243.

[13] Kotecha, A., Elsheikh, A., Roberts, C. R., Zhu, H., Garway-Heath, D. F., 2006. Corneal thickness- and age-related biomechanical properties of the cornea measured with the ocular response analyzer. Investigative Ophthalmology and Visual Science 47 (12), 5337-5347.

[14] Lago, M., Martínez-Martínez, F., Rupérez, M., Monserrat, C., Alcañiz, M., 2012. A Study about Coefficients to Estimate the Error in Biomechanical Models Used to Virtually Simulate the Organ Behaviors. In: Proceedings of international conference on Medicine Meets Virtual Reality. Vol. 173. IOS Press, pp. 250-256.

[15] Lanchares, E., Calvo, B., Cristóbal, J. A., Doblaré, M., 2008. Finite element simulation of arcuates for astigmatism correction. Journal of Biomechanics 41 (4), $797-805$. 
[16] Luce, D. A., 2005. Determining in-vivo biomechanical properties of the cornea with an ocular response analyzer. Journal of Cataract and Refractive Surgery 31 (1), $156-162$.

[17] Martínez-Martínez, F., Rupérez, M., Martín-Guerrero, J., Monserrat, C., Lago, M., Pareja, E., Brugger, S., López-Andújar, R., 2013. Estimation of the elastic parameters of human liver biomechanical models by means of medical images and evolutionary computation. Computer Methods and Programs in Biomedicine 111 (3), 537 - 549.

[18] Martínez-Martínez, F., Rupérez, M. J., Martín-Guerrero, J. D., Monserrat, C., Pareja, E., Brugger, S., López-Andújar, R., 2013. Estimation of the biomechanical parameters of the human liver avoiding invasive measuring methods. In: Proceedings of the 19th Congress of the European Society of Biomechanics (ESB 2013).

[19] Nguyen, T. D., Jones, R. E., Boyce, B. L., 2008. A nonlinear anisotropic viscoelastic model for the tensile behavior of the corneal stroma. Journal of Biomechanical Engineering 130 (4).

[20] Pandolfi, A., Fotia, G., Manganiello, F., 2009. Finite element simulations of laser refractive corneal surgery. Engineering with Computers 25 (1), 15-24.

[21] Shah, S., Laiquzzaman, M., Bhojwani, R., Mantry, S., Cunliffe, I., 2007. Assessment of the biomechanical properties of the cornea with the ocular response analyzer in normal and keratoconic eyes. Investigative Ophthalmology and Visual Science 48 (7), 3026-3031.

[22] Studer, H., Larrea, X., Riedwyl, H., Büchler, P., 2010. Biomechanical model of human cornea based on stromal microstructure. Journal of Biomechanics $43(5), 836-842$.

[23] Wollensak, G., Spoerl, E., Seiler, T., 2003. Stress-strain measurements of human and porcine corneas after riboflavinultraviolet-a-induced crosslinking. Journal of Cataract and Refractive Surgery 29 (9), 1780 - 1785. 